\title{
A SUBSTANTIAL DUST DISK SURROUNDING AN ACTIVELY ACCRETING FIRST-ASCENT GIANT STAR
}

\author{
C. Melis ${ }^{1,3}$, B. Zuckerman ${ }^{1}$, Inseok Song ${ }^{2}$, Joseph H. Rhee ${ }^{1}$, and Stanimir Metchev ${ }^{1,4}$ \\ ${ }^{1}$ Department of Physics and Astronomy, University of California, Los Angeles, CA 90095-1547, USA; cmelis@ astro.ucla.edu \\ 2 Department of Physics and Astronomy, University of Georgia, Athens, GA 30602-2451, USA \\ Received 2008 October 23; accepted 2009 February 19; published 2009 April 28
}

\begin{abstract}
We report identification of the first unambiguous example of what appears to be a new class of first-ascent giant stars that are actively accreting gas and dust and that are surrounded by substantial dusty disks. These old stars, who are nearing the end of their lives, are experiencing a rebirth into characteristics typically associated with newborn stars. The F2-type first-ascent giant star TYC 41443292 is in a wide separation binary system with an otherwise normal G8 IV star, TYC 4144329 1. From Keck near-infrared imaging and high-resolution spectroscopy, we are able to determine that these two stars are $\sim 1$ Gyr old and reside at a distance of $\sim 550 \mathrm{pc}$. One possible explanation for the origin of the accreting material is common-envelope interaction with a low-mass stellar or substellar companion. The gaseous and dusty material around TYC 4144329 2, as it is similar to the primordial disks observed around young classical T Tauri stars, could potentially give rise to a new generation of planets and/or planetesimals.

Key words: binaries: close - circumstellar matter - infrared: stars - stars: evolution - stars: individual (TYC 4144 329 1, TYC 4144329 2)

Online-only material: color figures
\end{abstract}

\section{INTRODUCTION}

It is well understood that stars are born in dusty, gaseous cocoons. The material enshrouding the star eventually settles into a circumstellar disk in which planet formation is thought to occur on short timescales. By several Myr after the star is born the primordial material that surrounded it has been accreted by the star, removed from the stellar system, or accumulated into larger objects. Thus, the majority of main-sequence and firstascent giant stars that we see are only sometimes surrounded by dusty debris disks (e.g., Meyer et al. 2004; Chen et al. 2005; Beichman et al. 2005; Low et al. 2005; Kim et al. 2005; Rhee et al. 2007; Zuckerman et al. 1995; Fekel et al. 1996; Jones 2008) and virtually never surrounded by gaseous disks that are actively accreting onto the star.

Here we report the first unambiguous example of a dusty, actively accreting first-ascent giant star, TYC 41443292. Zuckerman et al. (2008) discuss the possibility that BP Psc, a star with bipolar outflows and an orbiting gaseous ring detected in $\mathrm{CO}$ emission, may be a first-ascent giant of the same class. However, lacking a known distance, it is not yet clear if BP Psc is truly a first-ascent giant star or, rather, a strange, isolated T Tauri star. The case of TYC 41443292 is much more solid, thanks to the existence of an ordinary subgiant companion, TYC 41443291 . In the following sections we discuss our observations, determination of the spectral types for both stars, initial recognition of the infrared excess around TYC 4144 329 2, evidence for accretion onto the dusty star, and finally a determination of the age of the binary system.

\section{OBSERVATIONS}

At Mauna Kea Observatory, we observed both components of the TYC 4144329 system using the NIRC2 near-infrared imaging camera on the Keck II Telescope and the HIRES

\footnotetext{
3 Spitzer VGSP Fellow.

4 Current address: Department of Physics and Astronomy, State University of New York, Stony Brook, NY 11794-3800, USA.
}

echelle spectrometer at Keck I (see Table 1). Our NIRC2 data were taken using the natural guide star adaptive optics system (Wizinowich et al. 2000) to look for any extended emission around TYC 4144329 2. Due to complications in our data sets from anisoplanatic degradation, we can only place a tentative limit on any extent of TYC 41443292 of $\lesssim 70$ mas. Future measurements that will compensate for these effects are planned. Variable adaptive optics corrections prevented flux calibration of the TYC 4144329 images with a flux standard star. To determine the flux for TYC 4144329 2, we assumed TYC $41443291 \mathrm{had}$ photospheric flux values and computed delta-magnitudes for individual images. The results are discussed in Section 3.2.

Spectra of TYC 41443291 and 2 were obtained with the HIRES (Vogt et al. 1994) spectrometer on two dates (see Table 1). Spectral data were reduced and extracted using the $M A K E E$ software package.

Low-resolution grating spectra were obtained for the TYC 4144329 system with the KAST Double Spectrograph at the Lick Observatory Shane $3 \mathrm{~m}$ Telescope. Data obtained on UT 2008 June 28 suffered from extinction due to smoke from nearby fires. Data were reduced and extracted using standard IRAF tasks.

\section{RESULTS}

\subsection{TYC 4144329 Stellar Parameters}

Our star of interest, TYC 4144329 2, is part of a visual double star system. Table 2 contains information about TYC 4144329 1, the optical primary star, and TYC 41443292 , the dusty star. We took special care to ensure that we correctly determined the spectral type of both stars and especially the luminosity class of TYC 41443291 . Following previous works (Gray 1989; Strassmeier \& Fekel 1990; Kovtyukh et al. 2006) we employ line-depth ratios to determine absolute temperature and, for TYC 4144329 1, luminosity class. Such line ratio diagnostics have been shown to be relatively insensitive to metallicity, rotation, and surface gravity effects (Gray 1994; Kovtyukh et al. 2003, 2006). 
Table 1

Observations Summary

\begin{tabular}{|c|c|c|c|c|c|c|c|}
\hline UT Date & Instrument & Conditions & Setup & Coverage & Resolution $^{\mathrm{a}}$ & $\mathrm{S} / \mathrm{N}^{\mathrm{b}}$ & $\lambda$ of $\mathrm{S} / \mathrm{N}^{\mathrm{c}}$ \\
\hline 2007 Jun 23 & NIRC2 & Clear & $0^{\prime \prime} 01 \mathrm{pixel}^{-1}$ & $\mathrm{JHK}_{\mathrm{s}} \mathrm{L}^{\prime} \mathrm{M}_{\mathrm{s}}$ & $\ldots$ & $\ldots$ & $\cdots$ \\
\hline 2007 Oct 25 & NIRC2 & Nonphotometric & 0!'01 pixel $^{-1}$ & $\mathrm{JHK}_{\mathrm{s}} \mathrm{L}^{\prime}$ & $\ldots$ & $\ldots$ & $\ldots$ \\
\hline \multirow[t]{2}{*}{2007 May 5} & HIRES & Clear & UV Collimator-Blue & $3100-5900 \AA$ & 40,700 & $100 / 100$ & $5850 \AA$ \\
\hline & & & UV Collimator-Red & $4100-7000 \AA$ & 39,600 & $100 / 100$ & $6700 \AA ̊$ \\
\hline 2008 Feb 14 & HIRES & Cloudy & UV Collimator & $3000-5900 \AA$ & 39,700 & $80 / 50$ & $5500 \AA ̊$ \\
\hline \multirow[t]{2}{*}{$2008 \operatorname{Mar} 11^{\mathrm{d}}$} & KAST & Cloudy & $830 / 3460$ & $3000-4550 \AA$ & $\sim 3.4 \AA$ & $35 / 35$ & $4000 \AA$ \\
\hline & & & $600 / 7500$ & $4500-7000 \AA$ & $\sim 4.5 \AA$ & $100 / 100$ & $6000 \AA$ \\
\hline \multirow[t]{2}{*}{2008 Jun $28^{e}$} & KAST & See text & $600 / 4310$ & $3300-5500 \AA$ & $\sim 6.5 \AA$ & 75 & $4800 \AA$ \\
\hline & & & $830 / 8460$ & $5500-7300 \AA$ & $\sim 3.4 \AA$ & 75 & $6000 \AA$ \\
\hline
\end{tabular}

Notes.

${ }^{a}$ Resolutions were measured from the FWHM of single arclines in our comparison spectra.

${ }^{\mathrm{b}}$ S/N for TYC 4144329 1/TYC 41443292 unless otherwise noted.

${ }^{\mathrm{c}}$ Wavelength where $\mathrm{S} / \mathrm{N}$ measurement was made in the spectrum.

' Observations used the 1". 0 slit and the $\mathrm{d} 46$ dichoric.

${ }^{\mathrm{e}}$ Observations used the 1". 0 slit and the d55 dichoric. This entry is for the comparison star, HD 171994.

Table 2

Parameters of the TYC 4144329 Binary Star System

\begin{tabular}{|c|c|c|}
\hline \multicolumn{3}{|c|}{ Individual Stars } \\
\hline Parameter & TYC 41443291 & TYC 41443292 \\
\hline R.A. (J2000) & 102310.57 & 102309.88 \\
\hline Decl. (J2000) & +613646.0 & +613643.5 \\
\hline Sp. type & G8 IV & F2 \\
\hline Vmag & 10.12 & 10.75 \\
\hline$T_{\text {eff }}(\mathrm{K})$ & 5014 & $7000 \pm 200$ \\
\hline pmRA $\left(\right.$ mas yr $\left.^{-1}\right)$ & $-1.7 \pm 2.8$ & $+2.4 \pm 2.9$ \\
\hline $\mathrm{pmDE}\left(\mathrm{mas} \mathrm{yr}^{-1}\right)$ & $-25.5 \pm 2.8$ & $-24.3 \pm 2.8$ \\
\hline $\mathrm{RV}\left(\mathrm{km} \mathrm{s}^{-1}\right)$ & $-27.48 \pm 0.18$ & $-27.52 \pm 1.35$ \\
\hline Li I $6710 \AA ̊$ EW (mÅ) & $<2$ & $<20$ \\
\hline$v \sin i\left(\mathrm{~km} \mathrm{~s}^{-1}\right)$ & 3 & 31 \\
\hline \multicolumn{3}{|c|}{ System } \\
\hline Distance from Earth & \multicolumn{2}{|c|}{$\sim 550 \mathrm{pc}$} \\
\hline Separation between stars & \multicolumn{2}{|c|}{ 5..5 (projected separation of $3025 \mathrm{AU}$ ) } \\
\hline$U V W$ space motions $\left(\mathrm{km} \mathrm{s}^{-1}\right)$ & \multicolumn{2}{|c|}{$+9.4,-71.3,+4.4$} \\
\hline$[\mathrm{Fe} / \mathrm{H}]$ & \multicolumn{2}{|c|}{-0.2} \\
\hline Age & \multicolumn{2}{|c|}{$930 \pm 140 \mathrm{Myr}$} \\
\hline
\end{tabular}

Notes. See the text for discussion of stellar parameters. Samus \& Durlevich (2004) detect variability in visual combined light measurements of this system. They report maximum and minimum $V$-band magnitudes of 9.8 and 10.6, respectively. Similarly, combined light measurements of this system in the TASS Mark IV catalog (Droege et al. 2006) show changes in brightness indicating that one or both of the stars are variable. Two epochs of our NIRC2 data (UT 2007 June 23 and UT 2007 October 25) combined with 2MASS measurements (UT 1999 November 14) indicate variability for TYC 41443292 in the $J$ band at the tenth-of-a-magnitude level, but no such variability at longer wavelengths $(H$, $K_{\mathrm{s}}$, or $\left.L^{\prime}\right)$.

\subsubsection{TYC 41443292}

An initial estimate of the temperature class of TYC 4144 3292 came from a comparison of our Keck HIRES spectra to similar resolution spectra of stars of known spectral type that are not known to have any surrounding dust (Figure 1). A rough comparison of different ions of the same atom (Fe I and Fe II in the case of Figure 1) allows us to determine that TYC 41443292 has a temperature similar to that of an F2-type star $\left(T_{\text {eff }} \sim 7000 \mathrm{~K}\right)$. We attempted to further refine this estimate with line-depth ratios. Unfortunately, the broadened lines of TYC 41443292 complicate such diagnostics which typi-

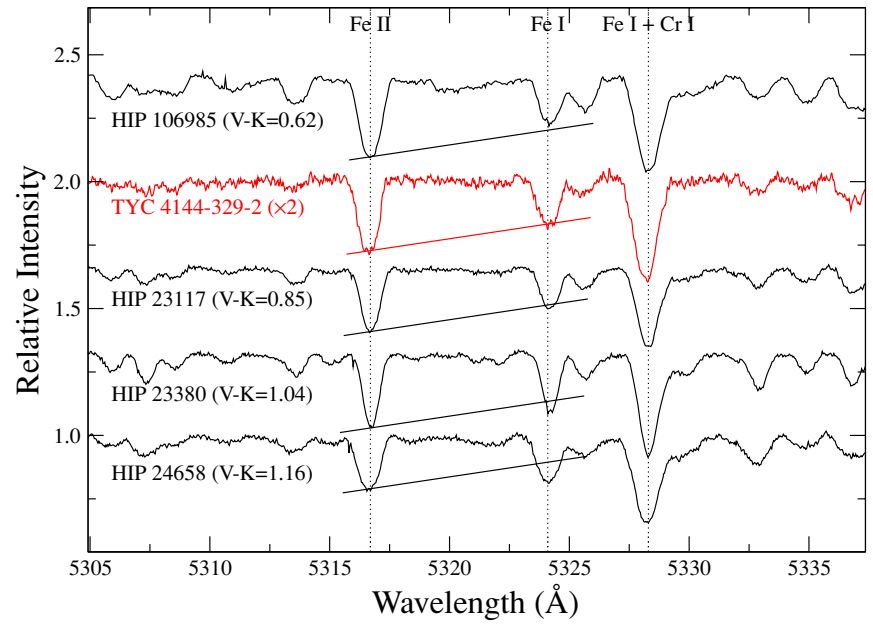

Figure 1. Keck HIRES spectra of TYC 41443292 (two individual exposures averaged together) and main-sequence, F-type stars not known to have any orbiting dust (the spectrum of HIP 106985 comes from the ELODIE archive; Moultaka et al. 2004). Comparison of temperature-sensitive absorption lines indicates that the temperature class of TYC 41443292 is somewhere between an A7 star $(V-K=0.62)$ and an F5 star $(V-K=1.042)$. The slanted lines are added to help guide the eye and have their slopes fixed at the slope value determined from comparing the line strength ratios of the Fe II and $\mathrm{Fe}$ I absorption lines of TYC 41443292 . The best match appears to be around an F2 $\operatorname{star}(V-K=0.85)$. Wavelengths in this figure are plotted in the heliocentric air scale. The comparison spectra have been shifted to TYC 4144329 2's heliocentric reference frame.

(A color version of this figure is available in the online journal.)

cally rely on comparing a stronger absorption line with a weak absorption line. With a $v \sin i$ of $\sim 30 \mathrm{~km} \mathrm{~s}^{-1}$, the weak lines of TYC 41443292 get subsumed into the continuum. As such, the most accurate line-depth ratios are unavailable to this analysis, and the best we can do is confirm our estimated temperature with lower limits. From comparing the line-depth ratios of Ti I $\lambda 6126$ to Si I $\lambda 6155$ with Figure 2 of Kovtyukh et al. (2003), we find that TYC 41443292 must have a temperature greater than $\sim 6500 \mathrm{~K}$. For the line-depth ratio of V I $\lambda 5727$ to Si I $\lambda 5772$ we are able to barely detect both lines after smoothing the spectrum with an 11-pixel boxcar, although we do not feel the detection of $\mathrm{V}$ I is greater than $3 \sigma$ significance. If we assume a detection then we get an effective temperature of $\sim 6700 \mathrm{~K}$ when comparing to Figure 2 of Kovtyukh et al. (2003); if we 

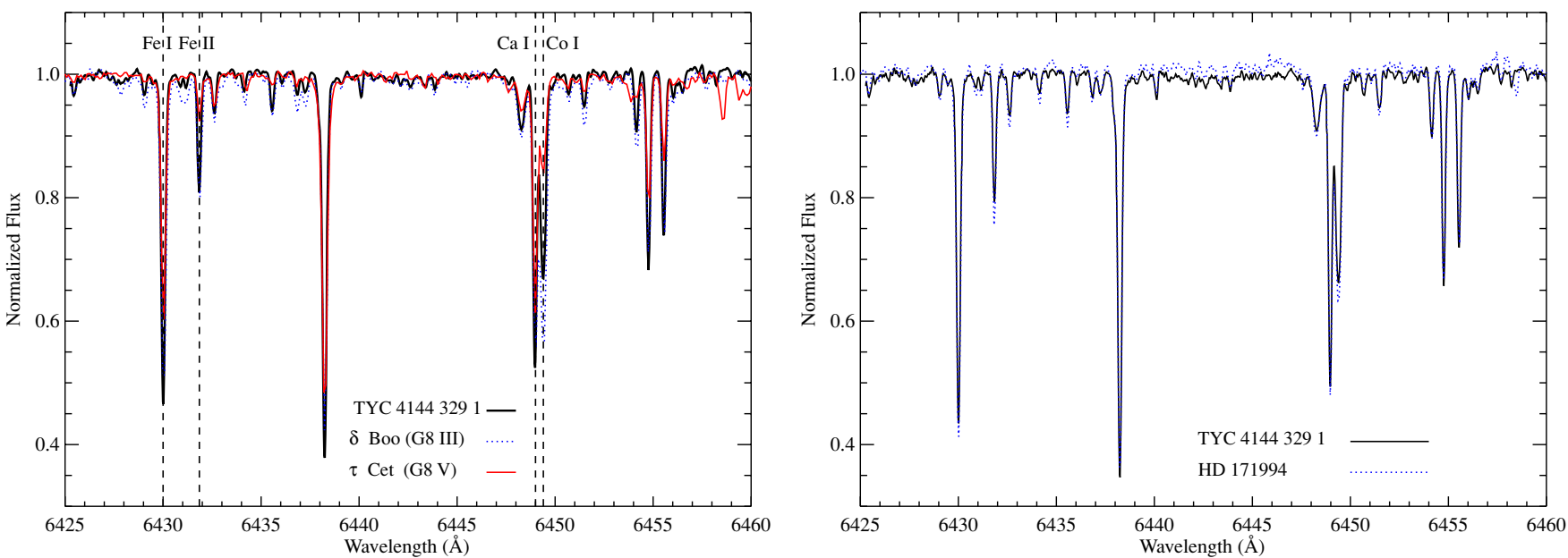

Figure 2. Both panels: comparison of our HIRES spectrum of TYC 41443291 with $\delta$ Boo, $\tau$ Cet, and HD 171994 (G8 III, G8 V, and G8 IV, respectively). All spectra presented in this figure have been shifted to the Earth rest frame with wavelengths in air. Left panel: luminosity class sensitive features (such as Co I $\lambda 6450$ ) indicate that TYC 41443291 has a luminosity class in between that of a giant star and a dwarf star (Strassmeier \& Fekel 1990). Right panel: TYC 41443291 with HD 171994 overplotted, both spectra have $R \sim 40,000$. A signal-to-noise ratio (S/N) $\sim 190$ HD 171994 spectrum was obtained from the ELODIE archive (Moultaka et al. 2004). We match our HIRES spectrum to the lower resolution pixel sampling of the ELODIE spectrum (pixel widths of $\sim 1.3 \mathrm{~km} \mathrm{~s}^{-1}$ and $\sim 3.1 \mathrm{~km} \mathrm{~s}{ }^{-1}$ for HIRES and ELODIE, respectively). HD 171994 is our qualitative best match to TYC 4144329 1, and hence we adopt its metallicity and absolute visual magnitude (Soubiran et al. 2008) for TYC 4144329 1. From the adopted absolute visual magnitude, we estimate a distance of $\sim 550 \mathrm{pc}$ to the TYC 4144329 system.

take the detection as an upper limit, the corresponding lower limit to the effective temperature of TYC 41443292 is $6700 \mathrm{~K}$. These two strong lower limits are consistent with our effective temperature estimate from comparison with nondusty field Ftype stars. As such, we feel confident that TYC 41443292 has a temperature class of $\mathrm{F} 2 \pm 2$ subclasses.

\subsubsection{TYC 41443291}

We make an initial estimate of the effective temperature of TYC 41443291 from broadband colors, finding a temperature class residing in the range of G8-K2 (the main uncertainty in this estimate is the luminosity class of TYC 41443291 ). With this estimate as a sanity check, we then employed line-depth ratios to refine the effective temperature measurement. Comparing the residual intensity ratio of $\mathrm{Fe}$ II $\lambda 6432$ to $\mathrm{Fe}$ I $\lambda 6430$ against Figure 2(a) of Strassmeier \& Fekel (1990) yields a temperature class of G8 \pm 1 subclass if TYC 41443291 is a giant or a subdwarf and $\mathrm{K} 0 \pm 1$ subclass if it is a dwarf. We employ additionally the line-depth ratios of $\mathrm{Fe}$ I $\lambda 6241$ to $\mathrm{Si}$ I $\lambda 6244$ and Fe I $\lambda 6704$ to Si I $\lambda 6722$ and find temperatures of $4800 \pm 100$ $\mathrm{K}$ for both when comparing against Figure 1 of Kovtyukh et al. (2006).

Our next step is to secure the luminosity class of TYC 4144 3291 and finalize its spectral type. As is illustrated in Figure 2, luminosity-sensitive lines (especially Co I $\lambda 6450$; Strassmeier \& Fekel 1990) show significant deepening with progression from dwarf to giant. Rough examination of Figure 2 shows that TYC 41443291 has a luminosity class intermediate to that of a dwarf and a giant star. Comparing the residual intensity ratio of Co I $\lambda 6450$ to $\mathrm{Ca}$ I $\lambda 6449$ with Figures 2(e) and 3(b) from Strassmeier \& Fekel (1990) shows good agreement with a subgiant classification. An additional check of the luminosity class of TYC 41443291 is obtained from comparing the flux ratio of bandpasses around $\mathrm{Fe}$ I $\lambda 4071$ and $\mathrm{Sr}$ II $\lambda 4077$ to Figure 1 of Mamajek et al. (2002). For our determined effective temperature, we find again that TYC 41443291 has line ratios consistent with a subgiant. With all of the above diagnostics, we are confident that the spectral type of TYC 41443291 is G8 IV.

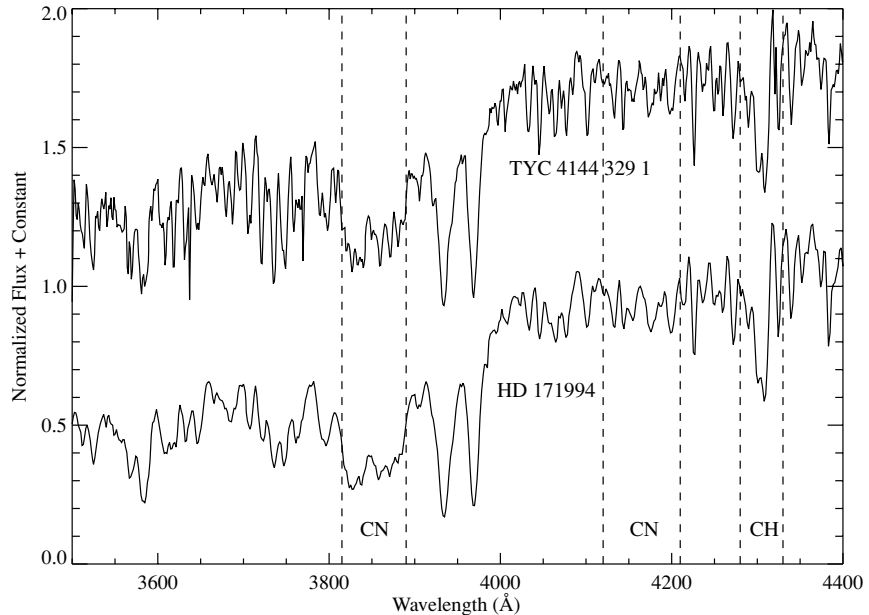

Figure 3. KAST blue side spectra of TYC 41443291 and HD 171994 with spectral resolutions of $\sim 3.4$ and $\sim 6.5 \AA$, respectively. Adverse conditions present during the acquisition of each spectrum (see Section 2 and Table 1) could have potentially affected the spectral calibrations. Although the spectral slopes are not identical, we find that line strengths, in particular the gravitysensitive $\mathrm{CN}$ and $\mathrm{CH}$ bands, are.

To complete the parameterization of TYC 41443291 we sought robust estimates of its metallicity and luminosity. In lieu of attempting to model our HIRES and KAST data we decided to qualitatively match our spectra with similar resolution spectra of stars with known metallicities and parallax measurements. Our best match to TYC 41443291 is HD 171994 (Figures 2 and 3). As such, we adopt the parameters of HD 171994 (see Soubiran et al. 2008) for TYC 41443291 (Table 2); specifically, the G8 IV spectral type classification, $T_{\text {eff }},[\mathrm{Fe} / \mathrm{H}]$, and the luminosity of HD 171994. The Hipparcos measured parallax of HD 171994 ( $\pi=10.70 \pm 0.44$ mas; van Leeuwen 2007) enables us to estimate a distance to TYC 41443291 . Based on the adopted absolute visual magnitude of 1.46 we estimate TYC 41443291 and TYC 41443292 (common space motions indicate the TYC 4144329 system is bound; see Table 2) reside at a distance of $\sim 550 \mathrm{pc}$. We conservatively adopt an uncertainty of $\sim 15 \%$ on this distance estimate. 


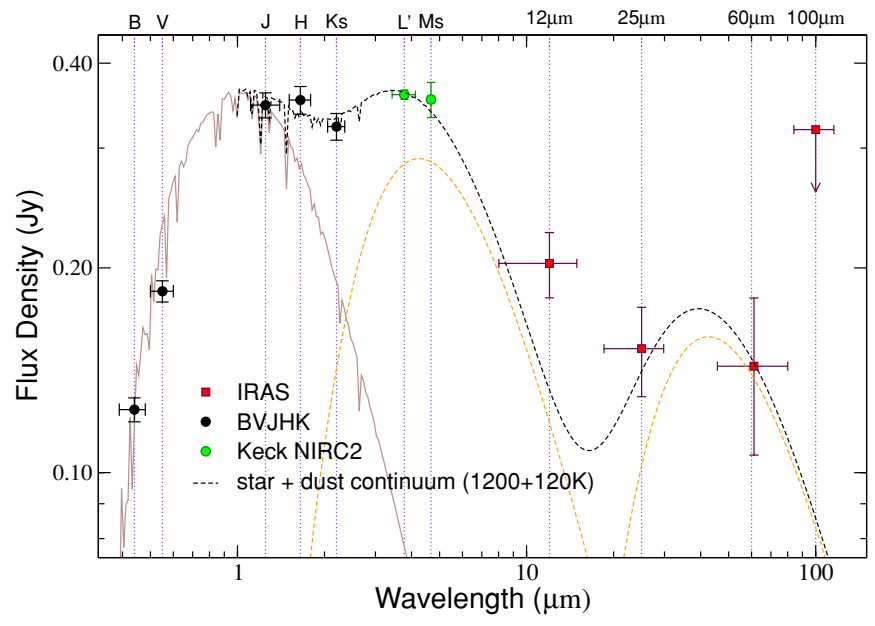

Figure 4. We detect significant infrared excesses from TYC 41443292 at wavelengths greater than $\sim 1 \mu \mathrm{m}$. The solid brown curve that peaks near $1 \mu \mathrm{m}$ is a synthetic stellar spectrum (Hauschildt et al. 1999) for a $7000 \mathrm{~K}$ effective temperature star reddened by an interstellar extinction model. The orange dashed curves that peak near $4 \mu \mathrm{m}$ and $40 \mu \mathrm{m}$ represent two dust continuum emission blackbodies fitted to dust temperatures of 1200 and $120 \mathrm{~K}$, respectively. The black dashed line, the sum of the above three curves, is a reasonable fit to most of the data points. The elevated position of the $12 \mu \mathrm{m}$ IRAS point suggests either a silicate emission feature or some dust with temperature intermediate between 120 and $1200 \mathrm{~K}$. The total luminosity of the excess, determined by integrating under the data points between $1 \mu \mathrm{m}$ and $100 \mu \mathrm{m}$, is $\sim 17 \%$ of the luminosity of the star. The BV data points (shortward of $1 \mu \mathrm{m}$ ) are from the $T Y C H O-2$ catalog, whereas the $\mathrm{JHK}_{\mathrm{s}}$ data points (longward of $1 \mu \mathrm{m}$ ) are from the 2MASS database. The green data points at $L^{\prime}$ and $M_{\mathrm{s}}$ are our own measurements obtained on UT 2007 June 23 with the Keck NIRC2 camera. The $L^{\prime}$ flux error bar is smaller than the point size on the plot. The four red data points from 12 to $100 \mu \mathrm{m}$ are from the IRAS Faint Source Catalog. The horizontal bars indicate the filter bandwidths.

(A color version of this figure is available in the online journal.)

\subsection{Excess Infrared Emission from TYC 41443292}

TYC 41443292 first came to our attention as part of a survey to identify $\mathrm{TYCHO}-2$ main-sequence stars with excess emission at mid- and far-infrared wavelengths (C. Melis et al. 2009 , in preparation). To accomplish this we cross-correlated the TYCHO-2 catalog (Høg et al. 2000) with the Infrared Astronomical Satellite catalogs (IRAS). The IRAS measurements of the TYC 4144329 binary at 12, 25, and $60 \mu \mathrm{m}$ (Figure 4) indicate the presence of warm and cool dust around at least one of the two TYCHO-2 stars (the IRAS beam size is many times larger than the 5.'5 separation between the two stars). Subsequent investigation of near-infrared fluxes at higher spatial resolution in the Two Micron All Sky Survey (2MASS) catalog (Cutri et al. 2003) revealed a definite $K_{\mathrm{s}}$-band excess around TYC 4144329 2, the F2-type star, but not around the G8 IV optical primary. To ensure that the overall infrared excess was real and arising purely from TYC 41443292 we observed the two stars with NIRC2. The relative $L^{\prime}$ and $M_{\mathrm{S}}$ fluxes indicate that the near-infrared excess emission originates entirely from the F2 star. Hence, we attribute any longer wavelength excess emission (mid- and far-infrared) to the F2 star.

To estimate the dust temperature and the fraction of the stellar luminosity reradiated by the dust $\left(L_{\mathrm{IR}} / L_{\mathrm{bol}}\right)$ we fit optical and near-infrared measurements out to $J$ band with a synthetic stellar atmosphere spectrum (Hauschildt et al. 1999) reddened by an interstellar extinction model (see caption to Figure 4) along with two blackbodies (having temperatures of 1200 and $120 \mathrm{~K}$, see Figure 4) that model the dust excess. The blackbody grains with
Table 3

Model Isochrone Fitting Parameters

\begin{tabular}{|c|c|c|}
\hline Parameter & TYC 41443291 & TYC 41443292 \\
\hline \multicolumn{3}{|c|}{ Inputs } \\
\hline $\log \left(L / L_{\odot}\right)$ & $1.49 \pm 0.07$ & $1.62 \pm 0.10$ \\
\hline $\log \left(T_{\text {eff }} / \mathrm{K}\right)$ & $3.700 \pm 0.009$ & $3.845 \pm 0.012$ \\
\hline \multicolumn{3}{|c|}{ Outputs } \\
\hline$M_{*}\left(M_{\odot}\right)$ & $2.07 \pm 0.10$ & $2.06 \pm 0.11$ \\
\hline $\log (g)$ & $3.04 \pm 0.11$ & $3.46 \pm 0.08$ \\
\hline Age (Myr) & \multicolumn{2}{|c|}{$930 \pm 140$} \\
\hline
\end{tabular}

Notes. The range in $\log \left(T_{\text {eff }} / \mathrm{K}\right)$ corresponds to roughly $\pm 100 \mathrm{~K}$ for TYC 4144 3291 and $\pm 200 \mathrm{~K}$ for TYC 41443292 . We determine the range in $\log \left(L / L_{\odot}\right)$ for TYC 41443291 from photometric, temperature, and distance errors that are propagated into its luminosity. We adopt a wider range in $\log \left(L / L_{\odot}\right)$ for TYC 41443292 to accommodate the contributions from variability and accretion luminosity. We determined the luminosity of TYC 41443292 by matching the monochromatic $J$-band magnitude to a blackbody with effective temperature of $7000 \mathrm{~K}$ at a distance of $550 \mathrm{pc}$. We then increased this resulting luminosity by $17 \%$ to account for stellar radiation reprocessed into the infrared (see Figure 4) and again by a factor of 1.28 to compensate for extinction assuming there were interstellar medium-like grains in TYC 4144329 2's dusty disk. Masses and surface gravities were calculated by averaging the maximum and minimum values obtained from all solutions to the isochrone fits (the isochrone fit output for the surface gravity of TYC 41443291 is consistent with HD 171994's surface gravity from Soubiran et al. 2008, where HD 171994 is the comparison star from which we adopted the TYC 41443291 input parameters).

a temperature of $120 \mathrm{~K}$ will reside at a distance of $\sim 30 \mathrm{AU}$ from TYC 41443292 . This distance of $30 \mathrm{AU}$ is derived under the assumption that TYC 41443292 has the luminosity listed in Table 3. Such a physical separation agrees well with the $\sim 45$ AU distance to similar temperature dust orbiting the lithiumrich giant star HD 233517 (Jura 2003a). Although the simple assumption of two narrow rings of dust provides a reasonable fit to the data, such a model is unlikely to be realistic considering that $\sim 17 \%$ of the total luminosity of the star is absorbed and reradiated at infrared wavelengths. To have so much light from the star processed by dust it is necessary to have an optically, vertically thick (e.g., flared) disk or a shell of dusty material surrounding the star.

\subsection{Accretion Signatures}

The optical spectrum of TYC 41443292 displays an uncharacteristically broad and deep He I $\lambda 5877$ absorption line (Figure 5). Although He I $\lambda 5877$ absorption lines are common in early- to mid-F type stars, they are typically fairly weak (Figure 5). The atmospheres of young $\mathrm{T}$ Tauri stars, which are far too cool to excite this helium absorption line, often show such a feature due to superheated regions of accreting disk gas (Alencar \& Basri 2000). Such a phenomenon could be boosting the strength of the helium absorption line in TYC 41443292. According to White \& Basri (2003), classical T Tauri stars with an $\mathrm{H} \alpha$ emission line $10 \%$ power width of $270 \mathrm{~km} \mathrm{~s}^{-1}$ or greater are accreting. The H $\alpha$ complex in TYC 41443292 shows both deep central absorption and double-peaked emission (Figure 5). Such double-peaked $\mathrm{H} \alpha$ emission lines are common in T Tauri stars, who are known to have substantial gaseous disks in orbit around them. Three methods of measuring the $\mathrm{H} \alpha$ emissionline width at $10 \%$ of peak power (from $10 \%$ of the maximum flux in Figure 5, from 10\% of the maximum of a parabolic fit anchored at the two emission peaks, and from $10 \%$ of a Gaussian anchored to the blue edge of the emission feature) yield an 

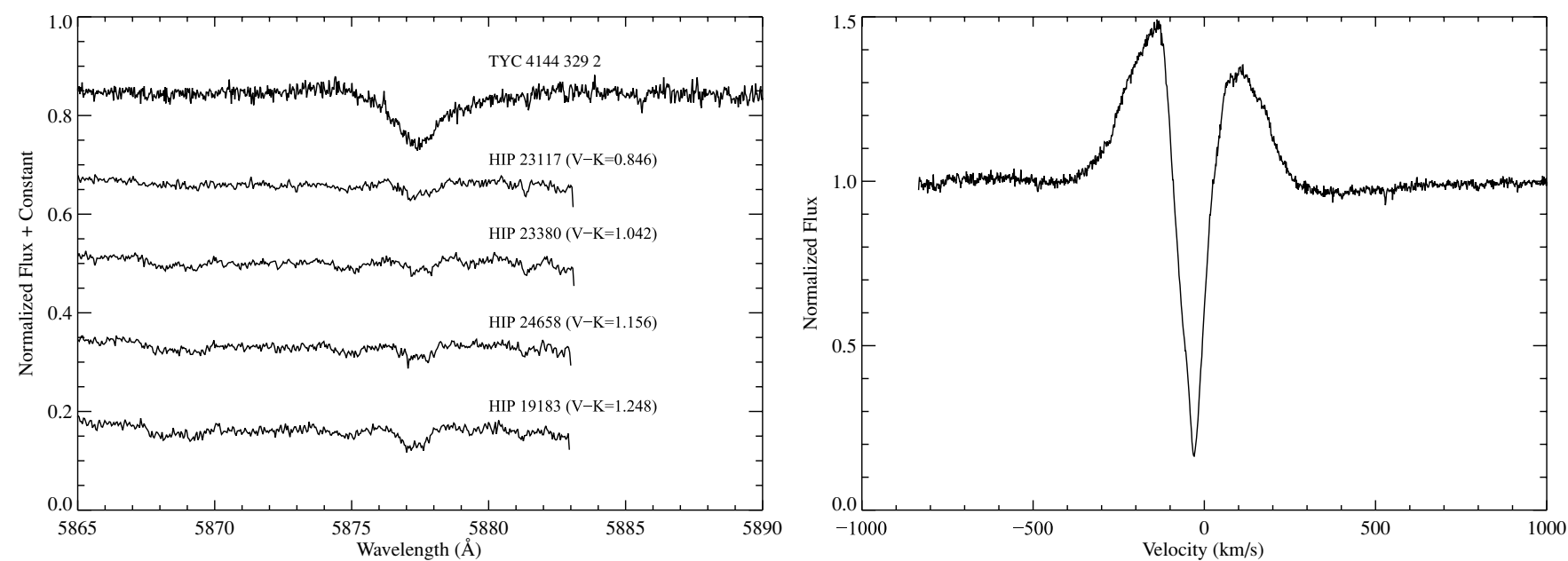

Figure 5. Left panel: Helium $\lambda 5877$ absorption feature in TYC 41443292 and nondusty main-sequence field F-type stars. These field F-type stars have He I $\lambda 5877$ EWs of $\sim 40 \mathrm{~m} \AA$, while TYC 41443292 has an He I $\lambda 5877$ EW of $\sim 290 \mathrm{~m} \AA$. Such an enhanced He I absorption feature could be the result of superheated accreting material (Alencar \& Basri 2000). Right panel: H $\alpha$ complex in TYC 4144329 2. The broad velocity width of the emission feature is consistent with a star that is accreting gas (White \& Basri 2003). The deep central absorption component might in part be due to disk gas orbiting the star. Similar features are seen in the $\mathrm{H} \beta$ absorption line and the $\mathrm{Na} \mathrm{D}$ absorption lines (see Section 3.3). Wavelengths in this figure are plotted in the heliocentric vacuum scale.

average of $556 \mathrm{~km} \mathrm{~s}^{-1}$ with a standard deviation of $30 \mathrm{~km} \mathrm{~s}^{-1}$. Employing Equation (1) from Natta et al. (2004) we find a mass accretion rate from the $\mathrm{H} \alpha 10 \%$ width of $10^{-7.5} M_{\odot} \mathrm{yr}^{-1}$. While characteristic of classical T Tauri stars, no such rapid accretion has ever been previously reported for a first-ascent giant star. To the best of our knowledge there are no known published reports of mass accretion at any rate, large or small, around such stars. ${ }^{5}$ We estimate the accretion luminosity for TYC 4144 3292 by comparing our determined accretion rate to young stars known to be accreting at the same rate with measured accretion luminosities (Muzerolle et al. 1998). From this method, we estimate the accretion luminosity for TYC 41443292 to be $\sim 0.1 L_{\odot}$, negligible compared to the stellar luminosity of TYC 41443292 .

There are shell-like absorption line components in the $\mathrm{Na}$ $\mathrm{D}, \mathrm{H} \alpha$, and $\mathrm{H} \beta$ profiles. The deep $\mathrm{H} \alpha$ absorption component (Figure 5) is blueshifted relative to the stellar velocity by $\sim 2.5 \mathrm{~km} \mathrm{~s}^{-1}, \mathrm{H} \beta$ has a similar velocity shift although there is unidentifiable structure present that is blended with the line of interest preventing a velocity measurement. The narrow components of the $\mathrm{Na} \mathrm{D}$ lines are blueshifted with respect to the stellar velocity by $\sim 1.5 \mathrm{~km} \mathrm{~s}^{-1}$ and they are just resolved (FWHM $\sim 8 \mathrm{~km} \mathrm{~s}^{-1}$ ) by HIRES (see Section 3.4). Such characteristics could be indicative of orbiting gas that is slowly moving away from the star. Similar absorption features in the debris-disk system around $\beta$-Pictoris were attributed to outer $\mathrm{Na}$-rich regions of the disk, although the $\mathrm{Na}$ lines in $\beta$-Pictoris are not shifted in radial velocity relative to the star (VidalMadjar et al. 1986). The narrow component of the $\mathrm{Na} \mathrm{D}_{2}$ line in $\beta$-Pictoris has an equivalent width (EW) of $9.5 \pm 1 \mathrm{~m} \AA$, while TYC 41443292 has a narrow $\mathrm{Na} \mathrm{D}_{2} \mathrm{EW}$ of $120 \pm 2 \mathrm{m \AA}$.

\subsection{Age of the TYC 4144329 System}

To aid in the determination of the age of the binary system, we obtained and analyzed high-resolution echelle spectra for both stars. From these optical spectra we estimate the age from the lithium content in the stellar photospheres, velocity

\footnotetext{
5 As mentioned in the Introduction, BP Psc may be a late-G first-ascent giant star (Zuckerman et al. 2008) with a mass accretion rate similar to that of TYC
} 41443292 widths of absorption lines, and Galactic space motion; details can be found in Zuckerman \& Song (2004). Of course, lithium content and velocity widths are best used to tell if stars are particularly young. In the following analysis, we only check that these parameters are consistent with our G8 IV spectral type of the optical primary. The lithium $6710 \AA$ absorption feature (wavelength in vacuum) is too weak to be detected in the spectrum of either star (Table 2). Lithium content in a stellar atmosphere is mainly determined by the star's age and mass. These upper limits are consistent with roughly solar mass stars at the end of their main-sequence lifetimes. Similarly, the low measured $v \sin i$ for TYC $41443291, \sim 3 \mathrm{~km} \mathrm{~s}^{-1}$, is consistent with an evolved star (de Medeiros \& Mayor 1995; Gray \& Pallavicini 1989; Schrijver \& Pols 1993). $v \sin i$ was measured from the full width at half-maximum (FHWM) depth of single absorption lines in the Keck HIRES spectra, which have intrinsic FWHM resolution of $\sim 8 \mathrm{~km} \mathrm{~s}^{-1}$, a value we subtract in quadrature from the FWHM measured in the spectra. A ROSAT All-Sky Survey nondetection of these stars is consistent with old stellar ages (Zuckerman \& Song 2004).

The above considerations suggest that the system is not young, in agreement with our spectral type determination of G8 IV for TYC 4144329 1. As a further refinement of our age estimate we can compare the space motions of the TYC 4144329 system with those of young stars and old white dwarfs (Figure 6). Velocities of the TYC 4144329 system toward the center of our Milky Way galaxy, around the Galactic center, and perpendicular to the Galactic plane $(U, V, W)$ are calculated from $T Y C H O-2$ proper motions, our estimated distance to the G8 IV star (see caption to Figure 2 and discussion in Section 3.1), and the optical echelle measured radial velocities. As stars age, the Galactic potential acts to disperse them in the $U-V$ and $U-W$ space. At a distance of $\sim 550 \mathrm{pc}$, the TYC 4144 329 system comfortably lies within the typical space motions of old white dwarf stars that have a mean cooling age of 1.86 Gyr (Figure 6; Zuckerman et al. 2003; Bergeron et al. 2001, note that the cooling age does not include the mainsequence lifetime, which is typically $\sim 1-3$ Gyr for these white dwarfs).

Finally, we determine more precisely the binary system's age by putting TYC 41443291 and 2 on theoretical isochrones 


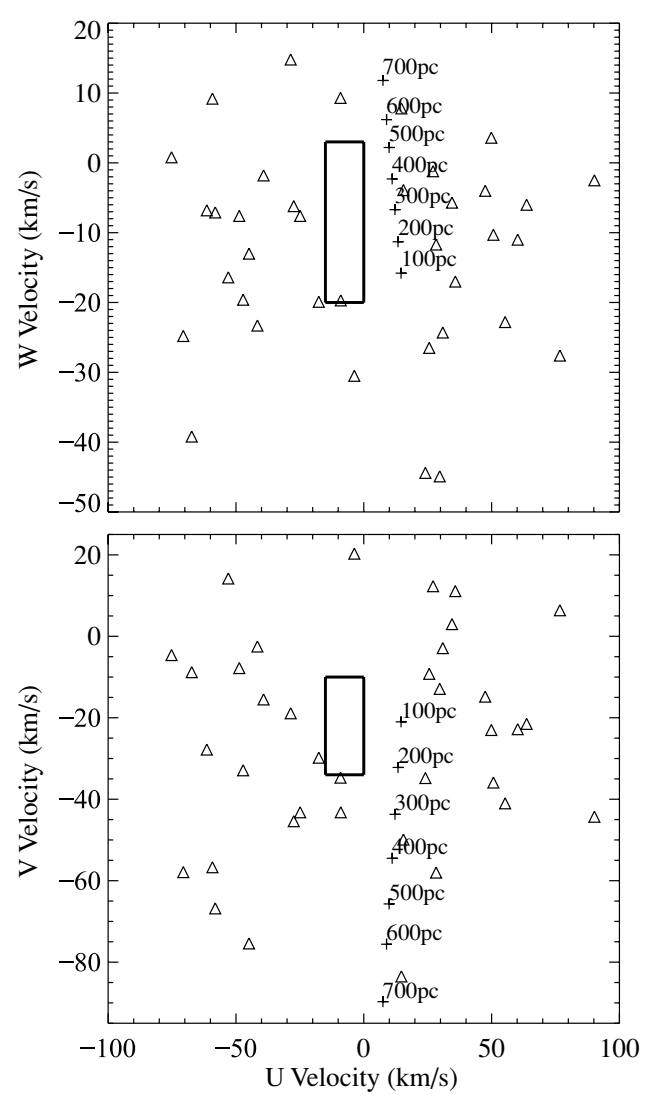

Figure 6. Comparison of the $U V W$ space motions with respect to the Sun for the TYC 4144329 system (crosses) with the $U V W$ of stars with known ages. We plot the entire range of plausible distances for the TYC 4144329 system as determined by ranging the luminosity of TYC 41443291 from that of a main-sequence dwarf to that of a class III giant. Triangles in the plot are white dwarfs with a mean cooling age of 1.86 Gyr (where the cooling age does not include the main-sequence lifetime of $\sim 1-3$ Gyr for the typical white dwarf in this sample; Zuckerman et al. 2003; Bergeron et al. 2001). The rectangles represent the "good" $U V W$ box for young stars as defined in Zuckerman \& Song (2004). As stars age, the Galactic potential acts to disperse them away from the young star boxes.

for evolved stars. We employed the CMD $2.1^{6}$ webform developed by Léo Girardi (Girardi et al. 2000; Marigo \& Girardi 2007; Bertelli et al. 1994; Marigo et al. 2008) to give model isochrones tailored to specific input metallicities. For our system metallicity of $[\mathrm{Fe} / \mathrm{H}] \sim-0.2$, we simultaneously fit the derived luminosities and effective temperatures for TYC 4144 3291 and 2 to the model isochrone. We list in Table 3 the input parameters and ranges for these values that were used for the model isochrone fit. From this fit, we determine that the TYC 4144329 system is $930 \pm 140 \mathrm{Myr}$ old. We note that for all acceptable ages obtained in this fashion TYC 41443291 and TYC 41443292 have evolved off the main sequence (see Figure 7).

Our above age determination suffers from a degeneracy between the size of the dust grains and the luminosity of TYC 4144329 2. In our analysis, we have assumed that the dust grains are interstellar-like as there is not yet any evidence for grain growth beyond that size. It is plausible that the grains in the disk surrounding TYC 41443292 could be larger. Larger grains would extinct the star more, and thus our derived luminosity would need to be increased appropriately (see note to Table 3 for how we estimated the luminosity of TYC 41443292 assuming interstellar-like dust grains extinct the star). However,

\footnotetext{
6 http://stev.oapd.inaf.it/cmd
}

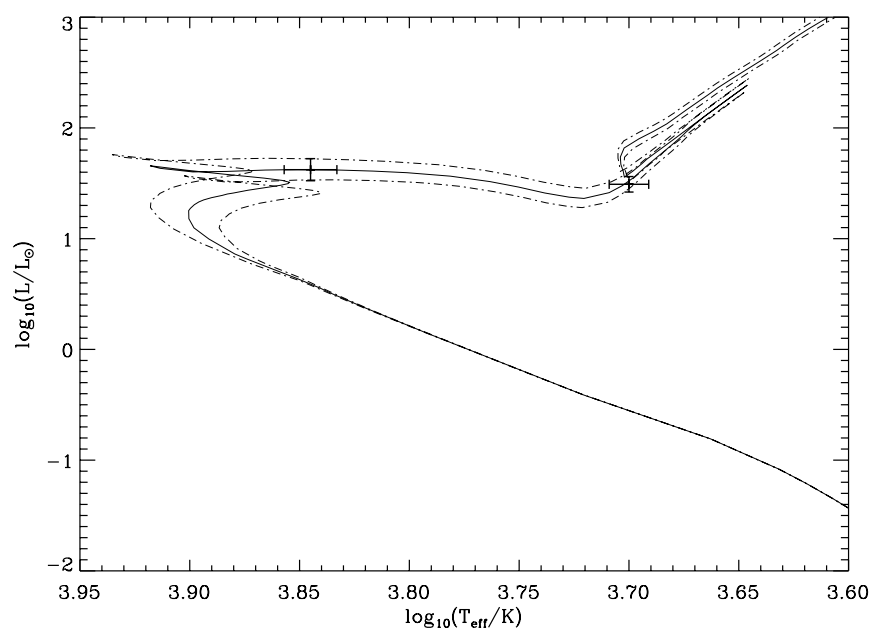

Figure 7. TYC 4144329 system plotted on theoretical isochrones. Isochrones were obtained from the CMD 2.1 webform (http://stev.oapd.inaf.it/cmd) and all have metallicity of $[\mathrm{Fe} / \mathrm{H}]=-0.2$. The solid isochrone represents our best-fit age for the TYC 4144329 system: $930 \mathrm{Myr}$. The two dot-dashed isochrones are for ages of $794 \mathrm{Myr}$ and $1027 \mathrm{Myr}$. TYC 41443291 is the point near $T_{\text {eff }}$ of 3.7 and $L$ of 1.5 , while TYC 41443292 is the point near $T_{\text {eff }}$ of 3.85 and $L$ of 1.6. Exact values of effective temperature and luminosity and their associated errors can be found in Table 3 .

there is a maximum grain size within the disk that is set by the necessity that both TYC 41443291 and 2 have the same age and thus can be fit simultaneously to a single isochrone. To estimate this maximum grain size, we examined the range of TYC 41443292 luminosities (with all other parameters held fixed) that still allow a simultaneous isochrone solution. The measured reddening, $E_{B-V}=0.32$, translates to $R=3.2$ for the interstellar-like grain scenario $\left(A_{V}=1.0\right)$, where $R$ is the ratio of total to selective extinction. The maximum luminosity TYC 41443292 can have and still allow an isochrone solution, $\log \left(L_{2} / L_{\odot}\right)=1.78$, corresponds to $R=4.0\left(A_{V}=1.3\right)$, suggesting grains slightly larger than interstellar dust. If the dust grains are this large then the system could be as young as 725 Myr old.

\section{DISCUSSION}

Based on the disk constituents, we postulate that the material accreting onto TYC 41443292 originated from an object composed mostly of gas. The TYC 4144329 system resides at high galactic latitude and no known interstellar molecular clouds are nearby. Potential sources of large quantities of gas would include the atmosphere of TYC 41443292 itself or a low-mass stellar or substellar companion, possibly even a massive Jupiter from the star's own planetary system. Main-sequence stars with companions at small separations are known: the W UMa class of main-sequence contact binaries and the "hot Jupiter" class of massive planets. When the F2 star evolved off the main sequence, there could have been interactions between it and its hypothetical short-orbital period companion (second epoch radial velocity measurements show no evidence for a remnant short-orbital period companion around TYC 4144329 2) that could create a circumstellar disk. There are plausible models of the aftermath of the engulfment of a short-period companion by a first-ascent giant star that results in the formation of a disk (Jura 2003a; Nordhaus \& Blackman 2006) as well as theoretical predictions of W UMa stars' endstates that involve the lower-mass companion transferring mass onto the evolved star 
and the formation of a circumbinary ring (Webbink 2003; Shu et al. 1979).

Evidence in support of a companion "consumption" model comes from two independent studies. The first is that of Tokovinin et al. (2006), in which the frequency of tertiary companions to spectroscopic binaries with short periods $(P<$ 30 days) is examined. They find that for very short orbitalperiod spectroscopic binaries $(P<2.9$ days $)$ the incidence of tertiary components is $\sim 100 \%$ (Figure 14 of Tokovinin et al. 2006). Indeed, the hypothetical companion that was consumed by TYC 41443292 must have been very shortperiod, as TYC 41443292 has only barely passed beyond main-sequence core hydrogen burning. The existence of TYC 41443291 (whose separation of $\sim 3000$ AU is consistent with the separations of the tertiaries studied by Tokovinin et al. 2006) is in accordance with such a scenario. A second observational campaign includes recent work on binary post-AGB stars. It has been revealed that these objects, when they host near-infrared excesses, are systematically found to be associated with binarity (de Ruyter et al. 2006). Confirmation that these near-infrared excesses are from Keplerian, circumbinary disks has come from interferometric observations of nearby systems (Bujarrabal et al. 2007; Deroo et al. 2007b, 2007a, and references therein). In these post-AGB systems, the binarity is seen as a likely necessity for the formation of a disk around the evolving giant star and its companion. The case for severe binary interaction, the type of which we assume occurred for TYC 41443292 but at a much earlier phase of its post-main-sequence evolution, is quite strong for these post-AGB stars (Deroo et al. 2007a).

We can make an estimate of the timescale of this phenomenon by noting that from of order $10^{5}$ stars in our TYCHO-2/IRAS survey who could have shown $L_{\mathrm{IR}} / L_{\mathrm{bol}} \sim 17 \%$, TYC 4144 3292 is the only such detection. Assuming that this system is $\sim 1$ Gyr old and that the phenomenon shows in one in $10^{5}$ stars, we estimate that the phenomenon might last of order $10^{4}$ years. We note that this timescale assumes that all stars of the input class (in this case first-ascent giant stars) at some time exhibit the dust and accretion features that we see in TYC 4144 329 2. If we instead assume our above companion consumption model is correct, and that the rate of binaries close enough to be consumed is $\sim 2 \%$ (Duguennoy \& Mayor 1991), then this timescale would be $\sim 10^{6}$ yr.

Lithium content in the F2 star's photosphere can be used to constrain the nature of the object that is the source of the accreting material. Brown dwarfs with mass $\lesssim 0.06 M_{\odot}$ should have a cosmic abundance of lithium as they never reach sufficient temperatures to burn it (Basri 2000). A low-mass star, however, would have burned all of its lithium by the time it is $\sim 1$ Gyr old (Zuckerman \& Song 2004). Lithium depletion in the atmosphere of main sequence, early F-type stars is a slow process that becomes even more inefficient as the star ages and spins down (Talon \& Charbonnel 1998). The timescale of substantial lithium depletion, on the order of several hundred Myr (Talon \& Charbonnel 1998), is much longer than our estimates of the timescale of the phenomenon occurring at TYC 41443292 . Thus, if any brown dwarf with a cosmic abundance of lithium had been accreted onto the photosphere of the F2 star we would definitely see a large EW lithium feature in our spectra. The fact that TYC 41443292 has begun to evolve off the main sequence does not affect this statement, as for any reasonable initial mass of TYC 41443292 a negligible amount of mass will be contained in the evolved star's slowly rotating convective envelope (see Mengel et al. 1979, Figure 1(e) and
Table 3). Since we do not see such a lithium feature, and because we know that any accreted object must have a substantial gas mass, we can safely rule out any accreted companion with a mass $<0.06 M_{\odot}$. It is also possible that the material in orbit around TYC 41443292 originated from itself; for example, the star's atmosphere may have been ejected by interactions with a common-envelope companion. In such a scenario, it is not possible to constrain the mass of the companion.

In closing we note one final implication of the nature of the material surrounding TYC 41443292 . For such optically thick material, a flat dust disk with an inner radius of order 30 stellar radii (determined from the hot component of the spectral energy distribution; Figure 4) cannot absorb 17\% of TYC 4144 329 2's luminosity (Jura 2003b). A disk must be puffed up or warped (Pringle 1996) to have the right geometry to process this amount of radiation. It is perhaps possible that a remnant gasgiant planet survived the event that occurred at TYC 41443292 and its orbit through the dusty and gaseous disk gravitationally perturbs the disk material. In addition, a current generation of planetesimals may be forming in the gaseous and dusty material as is expected for similar disks found around T Tauri stars. If this were the case, then hypothetical in situ radioactive dating of rocky objects around TYC 41443292 at some point in the future could reveal two significantly different ages, corresponding to the two epochs of planet formation that may have occurred in this system. Such a situation would be markedly different from that in the present-day Solar system, where the bulk of the asteroidal material is thought to have formed approximately contemporaneously with the Sun.

We are indebted to Frank Fekel for providing advice and comparison spectra to aid our determination of the spectral type of TYC 4144329 1. We thank Jason X. Prochaska and Michael Murphy for obtaining the UT 2008 March 11 KAST spectra for us. We thank Bruce Draine for kindly providing us with reddening models tailored to our data. We thank Bruce Macintosh and Christian Marois for obtaining one epoch of NIRC2 AO data for us. We are grateful to Peter Eggleton for helpful advice. Support for S.A.M. was provided by NASA through the Spitzer Fellowship Program, under award 1273192. The data presented herein were obtained at the W. M. Keck Observatory, which is operated as a scientific partnership among the California Institute of Technology, the University of California, and the National Aeronautics and Space Administration. The Observatory was made possible by the generous financial support of the W. M. Keck Foundation. This work was partially supported by the NASA ADP grant ADP06-0095 and by a NASA grant to UCLA. This research has made use of the VizieR service and of data products from 2MASS.

Facilities: IRAS, Keck:I(HIRES), Keck:II (NIRC2-NGSAO), Shane (KAST)

\section{REFERENCES}

Alencar, S. H. P., \& Basri, G. 2000, AJ, 119, 1881

Basri, G. 2000, ARA\&A, 38, 485

Beichman, C. A., et al. 2005, ApJ, 622, 1160

Bergeron, P., Leggett, S. K., \& Ruiz, M. T. 2001, ApJS, 133, 413

Bertelli, G., Bressan, A., Chiosi, C., Fagotto, F., \& Nasi, E. 1994, A\&AS, 106, 275

Bujarrabal, V., van Winckel, H., Neri, R., Alcolea, J., Castro-Carrizo, A., \& Deroo, P. 2007, A\&A, 468, L45

Chen, C. H., et al. 2005, ApJ, 634, 1372 
Cutri, R. M., et al. 2003, 2MASS All Sky Catalog of Point Sources The IRSA 2MASS All-Sky Point Source Catalog, NASA/IPAC Infrared Science Archive. http://irsa.ipac.caltech.edu/applications/Gator/

de Medeiros, J. R., \& Mayor, M. 1995, A\&A, 302, 745

de Ruyter, S., van Winckel, H., Maas, T., Lloyd Evans, T., Waters, L. B. F. M., \& Dejonghe, H. 2006, A\&A, 448, 641

Deroo, P., Acke, B., Verhoelst, T., Dominik, C., Tatulli, E., \& van Winckel, H. 2007a, A\&A, 474, L45

Deroo, P., van Winckel, H., Verhoelst, T., Min, M., Reyniers, M., \& Waters, L. B. F. M. 2007b, A\&A, 467, 1093

Droege, T. F., Richmond, M. W., Sallman, M. P., \& Creager, R. P. 2006, PASP, 118,1666

Duguennoy, A., \& Mayor, M. 1991, A\&A, 248, 485

Fekel, F. C., Webb, R. A., White, R. J., \& Zuckerman, B. 1996, ApJ, 462, L95

Girardi, L., Bressan, A., Bertelli, G., \& Chiosi, C. 2000, A\&AS, 141, 371

Gray, D. F. 1989, ApJ, 347, 1021

Gray, D. F. 1994, PASP, 106, 1248

Gray, D. F., \& Pallavicini, R. 1989, PASP, 101, 695

Hauschildt, P. H., Allard, F., \& Baron, E. 1999, ApJ, 512, 377

$\mathrm{H} ø$ g, E., et al. 2000, A\&A, 355, L27

Jones, M. H. 2008, MNRAS, 387, 845

Jura, M. 2003a, ApJ, 582, 1032

Jura, M. 2003b, ApJ, 584, L91

Kim, J. S., et al. 2005, ApJ, 632, 659

Kovtyukh, V. V., Mishenina, T. V., Gorbaneva, T. I., Bienaymé, O., Soubiran, C., \& Kantsen, L. E. 2006, Astron. Rep., 50, 134

Kovtyukh, V. V., Soubiran, C., Belik, S. I., \& Gorlova, N. I. 2003, A\&A, 411, 559

Low, F. J., Smith, P. S., Werner, M., Chen, C., Krause, V., Jura, M., \& Hines, D. C. 2005, ApJ, 631, 1170

Mamajek, E. E., Meyer, M. R., \& Liebert, J. 2002, AJ, 124, 1670

Marigo, P., \& Girardi, L. 2007, A\&A, 469, 239

Marigo, P., Girardi, L., Bressan, A., Groenewegen, M. A. T., Silva, L., \& Granato, G. L. 2008, A\&A, 482, 883
Mengel, J. G., Demarque, P., Sweigart, A. V., \& Gross, P. G. 1979, ApJS, 40, 733

Meyer, M. R., et al. 2004, ApJS, 154, 422

Moultaka, J., Ilovaisky, S. A., Prugniel, P., \& Soubiran, C. 2004, PASP, 116, 693

Muzerolle, J., Hartmann, L., \& Calvet, N. 1998, AJ, 116, 2965

Natta, A., Testi, L., Muzerolle, J., Randich, S., Comerón, F., \& Persi, P. 2004, A\&A, 424, 603

Nordhaus, J., \& Blackman, E. G. 2006, MNRAS, 370, 2004

Pringle, J. E. 1996, MNRAS, 281, 357

Rhee, J. H., Song, I., Zuckerman, B., \& McElwain, M. 2007, ApJ, 660, 1556

Samus, N. N., \& Durlevich, O. V. 2004, VizieR Online Data Catalog, 2250

Schrijver, C. J., \& Pols, O. R. 1993, A\&A, 278, 51

Shu, F. H., Anderson, L., \& Lubow, S. H. 1979, ApJ, 229, 223

Soubiran, C., Bienaymé, O., Mishenina, T. V., \& Kovtyukh, V. V. 2008, A\&A, 480,91

Strassmeier, K. G., \& Fekel, F. C. 1990, A\&A, 230, 389

Talon, S., \& Charbonnel, C. 1998, A\&A, 335, 959

Tokovinin, A., Thomas, S., Sterzik, M., \& Udry, S. 2006, A\&A, 450, 681

van Leeuwen, F. 2007, A\&A, 474, 653

Vidal-Madjar, A., Ferlet, R., Hobbs, L. M., Gry, C., \& Albert, C. E. 1986, A\&A, 167,325

Vogt, S. S., et al. 1994, Proc. SPIE, 2198, 362

Webbink, R. F. 2003, in ASP Conf. Ser. 293, 3D Stellar Evolution, ed. S. Turcotte, S. C. Keller, \& R. M. Cavallo (San Francisco, CA: ASP), 76

White, R. J., \& Basri, G. 2003, ApJ, 582, 1109

Wizinowich, P., et al. 2000, PASP, 112, 315

Zuckerman, B., Kim, S. S., \& Liu, T. 1995, ApJ, 446, L79

Zuckerman, B., Koester, D., Reid, I. N., \& Hünsch, M. 2003, ApJ, 596, 477

Zuckerman, B., \& Song, I. 2004, ARA\&A, 42, 685

Zuckerman, B., et al. 2008, ApJ, 683, 1085 